\title{
COMPORTAMENTO DO CONSUMIDOR E INTENÇÃO DE COMPRA DE SERVIÇOS HOTELEIROS: INFLUÊNCIA AO ASSISTIR VÍDEOS NA INTERNET
}

\author{
Marcela Martins Silva \\ Doutoranda e Mestre em Turismo \\ Universidade Federal do Rio Grande do Norte \\ mar_celams@hotmail.com \\ Jéssyca Rodrigues Henrique da Silva \\ Doutoranda e Mestre em Turismo \\ Universidade Federal do Rio Grande do Norte \\ jessyca.r.henrique@gmail.com \\ Sergio Marques Junior \\ Doutor em Agronomia e Professor do Programa de Pós-Graduação em Turismo \\ Universidade Federal do Rio Grande do Norte \\ sergio@ct.ufrn.br \\ Luiz Mendes Filho \\ Doutor em Administração e Professor do Programa de Pós-Graduação em Turismo \\ Universidade Federal do Rio Grande do Norte \\ luiz.mendesfilho@gmail.com
}

Resumo

Objetivo do estudo: Analisar o comportamento do consumidor no processo de compra de serviços hoteleiros turísticos ao assistir vídeos na internet.

Metodologia /abordagem: Utiliza-se o modelo proposto por Yang, Huang, Yang e Yang (2017), no qual comporta as atitudes do consumidor perante a anúncios na Web. Trata-se de um estudo de abordagem quantitativa, no qual os dados foram analisados por meio de análise fatorial confirmatória e matriz de correlação, utilizando o Software Statistical Package for the Social Sciences, versão 22.0 .

Originalidade/Relevância: Apesar dos estudos cada vez mais apostarem no uso de vídeos no contexto do turismo, pouco tem se analisado sobre o impacto destes veículos em organizações do setor. Este estudo amplia a pesquisa de Silva et al. (2018), no qual investigou o uso de vídeos no contexto de hotéis a partir da visão dos gestores, e, propõe uma nova abordagem a partir da percepção dos consumidores.

Principais resultados: A Informatividade e o Entretenimento foram as variáveis que mais se relacionaram positivamente. Por outro lado, a Irritação correlacionou-se negativamente com todas dimensões. Além disso, o estudo confirma que a capacidade de o telespectador entrar em Fluxo durante a exibição de um vídeo, contribui tanto para a Intenção, quanto para o Comportamento de compra.

Contribuições teóricas/metodológicas: Preenche lacuna acadêmica a respeito de pesquisas sobre comportamento do consumidor e o uso do marketing digital no setor hoteleiro através dos vídeos on-line, e sugere que os gestores dos hotéis invistam cada vez mais nestes mecanismos para melhoria de seu marketing na internet.

Palavras-chave: Marketing digital. Intenção de Compra. Serviços Hoteleiros. Vídeos.

\section{$\underline{\text { Cite como }}$}

American Psychological Association (APA)

Silva, M. M., Silva, J. R. H., Marques Junior, S., \& Mendes Filho, L. (2020). Comportamento do consumidor e intenção de compra de serviços hoteleiros: influência ao assistir vídeos na internet. PODIUM Sport, Leisure and Tourism Review, São Paulo, 9(2), 286-307.

https://doi.org/10.5585/podium.v9i2.15905. 
Silva, M. M., Silva, J. R. H., Marques Junior, S., \& Mendes Filho, L. (2020). Comportamento do consumidor e intenção de compra de serviços hoteleiros: influência ao assistir vídeos na internet

\title{
CONSUMER BEHAVIOR AND INTENTION TO PURCHASE HOTEL SERVICES: INFLUENCE WHEN WATCHING VIDEOS ON THE INTERNET
}

\begin{abstract}
Objective of the study: Analyze consumer behavior in the process of purchasing tourist hotel services when watching videos on the internet.

Methodology / approach: It was used the model proposed by Yang, Huang, Yang and Yang (2017), which includes consumer attitudes towards web advertisements. This is a quantitative approach study, in which data were analyzed by confirmatory factor analysis and correlation matrix using the Statistical Package for Social Sciences Software, version 22.0.

Originality / Relevance: Although studies increasingly focus on the use of videos in the context of tourism, little has been analyzed about the impact of them on the industry. This study broadens the research by Silva et al. (2018), in which it was investigated the use of videos in the context of hotels from the managers 'point of view, and proposes a new approach from the consumers' perception.

Main results: Informativity and Entertainment were the variables that most positively related. On the other hand, Irritation was negatively correlated with all dimensions. In addition, the study confirms that the ability of the viewer to enter the Stream during the display of a video, contributes to both the intention and the purchase behavior.
\end{abstract}

Theoretical / Methodological Contributions: It fills the academic gap regarding research on consumer behavior and the use of digital marketing in the hotel sector through online videos, and suggests that hotel managers increasingly invest in these mechanisms to improve their internet marketing.

Keywords: Digital Marketing. Buy intention. Hotel services. Videos.

\section{COMPORTAMIENTO DEL CONSUMIDOR E INTENCIÓN DE COMPRAR SERVICIOS DEL HOTEL: INFLUENCIA AL VER VIDEOS EN INTERNET}

\section{Resumen}

Objetivo del estudio: Analice el comportamiento del consumidor en el proceso de compra de servicios de hoteles turísticos cuando vea videos en Internet.

Metodología / enfoque: Utilizamos el modelo propuesto por Yang, Huang, Yang y Yang (2017), que incluye las actitudes de los consumidores hacia los anuncios web. Este es un estudio de enfoque cuantitativo, en el que los datos se analizaron mediante análisis factorial confirmatorio y matriz de correlación utilizando el paquete estadístico para software de ciencias sociales, versión 22.0.

Originalidad / relevancia: A pesar de que los estudios apuestan cada vez más por el uso de videos en el contexto del turismo, se ha analizado poco sobre el impacto de estos vehículos en las organizaciones del sector. Este estudio amplía el investigación de Silva et al. (2018), en el que investigó el uso de videos en el contexto de los hoteles desde el punto de vista de los gerentes, y propone un nuevo enfoque desde la percepción de los consumidores.

Resultados principales: La informatividad y el entretenimiento fueron las variables que se relacionaron más positivamente. Por otro lado, la irritación se correlacionó negativamente con todas las dimensiones. Además, el estudio confirma que la capacidad del espectador para ingresar al Stream durante la visualización de un video contribuye tanto a la intención como al comportamiento de compra. 
Contribuciones teóricas / metodológicas: Llena la brecha académica con respecto a la investigación sobre el comportamiento del consumidor y el uso del marketing digital en el sector hotelero a través de videos en línea, y sugiere que los gerentes de los hoteles están invirtiendo cada vez más en estos mecanismos para mejorar su marketing en Internet.

Palabras clave: Marketing digital. Intención de compra. Servicios hoteleiros. Videos.

\section{Introdução}

Com as mudanças advindas da globalização, avanços tecnológicos e dos meios de comunicação, o setor de marketing tem se reinventado e muitas das estratégias que antes eram adotadas no modelo tradicional, modificaram-se para compor um novo processo comunicacional pautado no ambiente on-line. Estudos mostram que nos próximos anos, 90\% do crescimento das empresas serão proporcionados por companhias que investem em estratégias de engajamento com os seus clientes (Administradores, 2017). Isto tem ocorrido, pois os meios de comunicação estão enfrentando um novo usuário digital, que se tornou um tipo de consumidor que participa, produz e requer uma comunicação diferente para que prendam sua atenção ao conteúdo divulgado (Montemayor \& Ortiz Sobrino, 2016). Portanto, o marketing digital se constitui como um meio para as empresas divulgarem seus produtos, serviços e marcas, no qual os canais de comunicação são utilizados na busca de uma vantagem competitiva.

Diante deste contexto, os vídeos publicados na internet têm chamado a atenção das pessoas, dos profissionais da área de marketing e dos acadêmicos, como uma estratégia eficiente para a comunicação e efetivação de vendas. Para Berthon, Pitt e DesAutels (2011), se o texto era o meio de comunicação da era analógica, o vídeo é hoje o meio utilizado na era digital. Esses mecanismos tornaram-se ferramentas de fácil compreensão para os usuários, permitindo permear por diferentes plataformas digitais, gerando impactos bilionários (Montemayor \& Ortiz Sobrino, 2016).

Pesquisas de tendências têm comprovado essa eficiência. Por exemplo, nos Estados Unidos, existe uma previsão que até o ano de 2021 os gastos com anúncios em vídeos sociais aumentarão $44 \%$ em relação ao ano de 2019, representando um total de 30,4\% do gasto total com anúncio. Já para 2023, estima-se que este percentual se eleve para 62,1\% (E-Marketer, 2019). Dessa forma, os profissionais de marketing devem investir no uso de vídeos em mídias sociais, pois a pesquisa realizada por Brightcove (2018), mostrou que estes são os canais 
preferidos pelas gerações mais jovens, os chamados Millennials ou geração Y (que são as pessoas nascidas no início da década de 1980) e os denominados geração Z (nascidos entre a metade dos anos de 1990 até o início do ano de 2010). Segundo Brightcove (2018), 66\% dos participantes dessas gerações afirmaram que se envolvem com uma marca após assistir um vídeo on-line.

Já o marketing turístico enfrenta desafios, pois diferentemente de outros setores industriais, no turismo não existe pré-teste, ou seja, uma forma do turista experimentar o produto antes da sua aquisição (Guimarães \& Borges, 2008). Por isto, este é um tema aguçado para o turismo e alguns estudos já têm demonstrado a influência do uso de vídeos no processo de decisão de compra de potenciais viajantes (Tussyadiah \& Fesenmaier, 2009; Reino \& Hay, 2011; Tsai, Chu \& Kobori, 2017; Amir, Pennington-cinzento, Barbe e Hanafiah, 2018; Silva, Silva \& Mendes Filho, 2018).

Além disso, a imagem possui o papel de mediadora da experiência turística e exerce influência na intenção de adquirir uma viagem, produto ou serviço turístico (Tussyadiah \& Fesenmaier, 2009; Reino \& Hay, 2011; Tsai et al., 2017). No entanto, o uso da imagem estática já não tem sido suficiente para o turista do mundo moderno estabelecer sua decisão, e por isso, o uso de vídeos tem ganhado visibilidade (Reino \& Hay, 2011). Eles podem instituir uma maior percepção de segurança, diminuir percepções negativas de medo e incentivar o turista a realizar a compra (Amir et al., 2018).

Apesar dos estudos cada vez mais apostarem no uso de vídeos no contexto da atividade turística, a maioria dos trabalhos concentram-se em avaliá-los durante a escolha de destinos turísticos e poucos analisaram o impacto destes veículos no cenário de organizações do setor. Sendo assim, considerando o papel que o setor hoteleiro exerce nas viagens e turismo, este estudo amplia o estudo de Silva et al. (2018), no qual os autores investigaram o uso de vídeos no contexto de empresas hoteleiras a partir da visão dos gestores, propondo uma nova abordagem por meio da avaliação da percepção dos consumidores, identificando os fatores que influenciam a intenção e comportamento de compra de serviços hoteleiros ao assistir vídeos na internet.

Entender como o vídeo afeta a intenção de compra do consumidor turístico se faz necessário para que ações e planejamentos sejam melhores direcionados. Neste sentido, este estudo tem como objetivo analisar o comportamento do consumidor no processo de compra de serviços hoteleiros turísticos ao assistir vídeos na internet. Para isto, a pesquisa baseou-se na 
metodologia proposta por Yang, Huang, Yang e Yang (2017) para investigar as atitudes do consumidor ao assistir vídeos on-line, e consequentemente, como alguns fatores afetam seu comportamento e o processo de compra.

Portanto, este estudo traz contribuições acadêmicas, além de implicações práticas e gerenciais. Para a academia o estudo é importante à medida que preenche uma lacuna a respeito das pesquisas sobre o uso do marketing digital no setor hoteleiro através dos vídeos on-line, e consequentemente, como esses recursos influenciam no comportamento do consumidor desses empreendimentos. Em relação às implicações práticas/gerenciais, a pesquisa fornece informações aos gestores de marketing sobre os fatores influenciáveis, ou não, do ponto de vista do consumidor, que servirão para elaborar campanhas de comunicação para o setor hoteleiro a partir desses recursos, ou seja, dos vídeos on-line.

A seguir apresenta-se a fundamentação teórica onde foram utilizados os conceitos de marketing digital, vídeos e intenção de compra no turismo.

\section{Marketing digital, vídeos e intenção de compra no turismo}

Com a popularização da internet, novas formas de comunicação emergiram, proporcionando diferentes possibilidades para a intermediação das relações entre fornecedor e cliente. Esse processo impõe ao setor do marketing, desafios em termos de renovação de suas estratégias, pois surgiram maneiras mais eficientes de levar a informação, persuadir e incentivar o cliente a realizar a compra. $\mathrm{O}$ marketing digital se caracteriza como uma alternativa para a realização do marketing comum, no qual pode incluir uma única estratégia, ou um conjunto delas utilizadas para impactar um público-alvo. Para isto, os profissionais de marketing utilizam alguns mecanismos tecnológicos, tais como, computador, mídias sociais on-line, websites, sites de buscas, notícias ou e-mails (Ogden \& Crescitelli, 2007). Montemayor e Ortiz Sobrino (2016, p. 88) fazem uma reflexão em relação à mídia social ao considerarem que ela está se reinventando e esclarecem que "vivenciamos uma mudança estrutural, movendo-se um paradigma dinâmico, onde a comunicação digital interativa é um espaço de convergência cultural e tecnológica, caracterizada pela presença de tecnologias que afetam a produção, distribuição e consumo de conteúdo".

Neste contexto, um dos recursos que tem ganhado visibilidade dentro do marketing digital são os vídeos publicados na internet. Os vídeos caracterizam-se como "parte substancial das interações que criam informação globalmente compartilhada, para o qual, de acordo com 
uma metáfora espacial chamamos ciberespaço, espaço navegável, espaço que podemos navegar, graças à linguagem de hipertexto (Díaz Arias, 2009, p. 64). Para Yang et al. (2017) propagandas através de vídeos on-line têm oferecido grandes oportunidades para as empresas. Eles têm servido como uma ferramenta de comunicação inovadora, pelo qual as publicidades das marcas estão inserindo suas mensagens na mente dos consumidores (Montemayor \& Ortiz Sobrino, 2016). Além disso, seus compartilhamentos bidirecionais permitem que não só as empresas compartilhem suas informações para os consumidores, mas também, que os próprios consumidores relatem suas experiências para as empresas e para outros consumidores (Berthon et al., 2011; Reino \& Hay, 2011, Silva, Mendes Filho e Marques Júnior, 2019).

No contexto da atividade turística, o marketing digital através do uso de vídeos vem ganhando visibilidade, pois a imagem é para o turismo uma ferramenta que permite uma comunicação mais eficiente, minimizando a percepção do risco na compra, por vezes, sentida pelo cliente. "Como os turistas estão cada vez mais interessados em experiências reais e autênticas em seu tempo de lazer, e como o tempo se torna mais espremido, eles buscam fontes de informações que vão o mais longe possível, para aumentar as suas experiências e reduzir suas chances de uma experiência decepcionante" (Reino \& Hay, 2011, p.3). Amir et al. (2018) argumentam que com a proliferação das redes sociais on-line as características audiovisuais durante a comunicação da mensagem por vídeos podem se constituir uma maneira eficiente para diminuir percepções negativas sobre um produto ou destino turístico.

Para Tussyadiah e Fesenmaier (2009), o marketing de destinos cada vez mais se apoia em recursos de imagens e vídeos em seus sites, para melhorar o apoio comunicacional com os turistas. Esses vídeos são utilizados como uma forma de intensificar o consumo de viajantes e são mostrados como uma poderosa mídia de "transporte" dentro do conceito de mobilidade virtual. Os autores afirmam que os vídeos afetam e possuem o papel de mediador da experiência turística, sendo capazes de gerar prazeres mentais, sonhos e fantasias que fazem o potencial turista visitar o destino. Como muitos consumidores não possuem tempo para assistir vídeos de longa duração, organizações nacionais de turismo em países como Coréia, Taiwan, Malásia e Índia, passaram a promover clips de vídeos com uma menor duração, para que os telespectadores possam assisti-los em menos tempo (Tsai et al., 2017).

Tsai e Bui (2020, p. 1) explicam que "nas últimas décadas, a mídia social evoluiu para vários serviços de rede social que permitem que usuários de todo o mundo interajam e compartilhem experiências de viagem por meio de postagens em texto, vídeos e fotografias". 
Neste contexto, o Conteúdo Gerado pelo Usuário (CGU) é definido como toda forma de conteúdo gerado por consumidores na internet (Litvin, Goldsmith, \& Pan, 2008, 2018). No turismo, o CGU tem sido extremamente útil para o planejamento de viagens, já que os turistas o utilizam para buscar informações sobre produtos e serviços turísticos que precisam consumir. Com o uso do CGU, os turistas estão se tornando cada vez mais empoderados no processo de planejamento de viagens (Mendes-Filho, Mills, Tan \& Milne, 2018). Logo, o consumidor do turismo tem elevado seus níveis de capacitação, se tornando cada vez mais habilitado para planejar suas viagens de forma livre e independente (Mendes Filho, 2014).

Dentro desse contexto, o CGU tem substituído o marketing boca-a-boca tradicional, para buscas de informações baseadas na Web. Isso porque mensagens baseadas em texto, vídeos e imagens, geram na internet um tipo de boca-a-boca on-line (Electronic Word of Mouth - eWOM) que auxiliam os turistas a se concentrarem em experiências relacionadas ao turismo e interagirem com os outros viajantes que consideram confiáveis (Tsai \& Bui, 2020). Litvin, Goldsmith e Pan (2018, p.314), conceituam o boca-a-boca on-line como a "variedade de maneiras pelas quais as pessoas comuns interagem on-line, por meio de blogs, comunidades virtuais, sites de revisão de produtos, e-mail, grupos de notícias, salas de bate-papo e mensagens instantâneas". Além disso, esses comentários têm influenciado significativamente a intenção, comportamento e decisão de compra dos clientes (Ramos, Mendes Filho \& Lobianco, 2017). No entanto, o boca-a-boca on-line pode ter uma influência tanto positiva, quanto negativa. Isso sugere que os profissionais de marketing não só devem incentivar os turistas a realizarem postagens positivas, como também devem encontrar estratégias para impedi-los de compartilhar sentimentos negativos (Yan, Zhou, \& Wu, 2018).

De acordo com Reino e Hay (2011), os turistas estão cada vez mais céticos para acreditar em algo que são passados a eles. Esse fato tem forçado as organizações a buscarem formas mais convincentes de vender seus produtos e serviços, sem despertar a desconfiança de seus consumidores. Por isto, novas estratégias de marketing em vídeos devem ser implementadas, seja na forma de incentivo aos clientes para produzirem o CGU, ou na utilização de provedores populares de mídias sociais para produzir vídeos para empresas do turismo.

O setor hoteleiro é um subsetor do turismo e para Silva et al. (2018), é importante que essas empresas invistam no preparo de profissionais para gerenciar o uso de vídeos no marketing digital dessas organizações. Esses autores ainda esclarecem que os vídeos devem ser publicados, não só no site oficial do empreendimento, mas também de forma espontânea, 
através de "lives" compartilhadas em redes sociais on-line. Também sugerem que os hotéis elaborem estratégias para incentivar os hóspedes a compartilharem suas experiências na internet, já que o conteúdo gerado por estes clientes, poderá repassar maior confiança sobre o produto ou serviço oferecido em seus estabelecimentos.

Diante do exposto, os vídeos se apresentam como uma tendência de marketing digital para o mundo moderno e dinâmico, e as organizações, sejam elas, públicas e privadas que não conseguirem se adaptar a esta realidade correm o risco de perder espaço no mercado.

\section{Metodologia}

\subsection{Modelo Teórico}

Yang et al. (2017) desenvolveram um modelo para analisar as atitudes dos consumidores perante as propagandas em vídeos on-line. O modelo é a extensão e integração do modelo Brackett e Carr (2001), da Teoria da Ação Racional e da Teoria do Fluxo. O estudo de Yang et al. (2017) foi realizado com estudantes e somente com uma plataforma de vídeo, o YouTube. Partindo da recomendação dos autores que sugerem a replicação da pesquisa em outra realidade mercadológica, a intenção desta pesquisa é examinar como o modelo proposto por Yang et al. (2017) se comporta na realidade do turismo, a partir da intenção de compra de serviços hoteleiros, e não somente em uma única plataforma, mas com uma abordagem mais abrangente no contexto da internet.

O modelo de pesquisa de Brackett e Car (2001) possui os constructos: entretenimento, informatividade, irritação e credibilidade. Esses constructos foram considerados preditores da atitude em relação aos anúncios em vídeos on-line. Assim, obtiveram resultados positivos suportados, confirmando a relação, além de fortalecer o modelo de Brackett e Car (2001).

O modelo de Brackett e Car (2001) é formado a partir de estudos de Ducoffe (1996), com os constructos: Entretenimento, Informatividade e Irritação, no qual verificou-se que eles afetavam as atitudes no contexto de anúncios e publicidade na internet. O resultado mostrou que a Informatividade e o Entretenimento são antecedentes significativos para se ter êxito com a publicidade na internet, sendo que a Irritação teve influência negativa nas atitudes. A partir de estudos de Eighmey (1997), também foi adicionado o constructo Credibilidade, no qual atestou uma relação direta e positiva com as atitudes dos consumidores em relação aos anúncios 
publicitários. As definições que Brackett e Car (2001) e Yang et.al. (2017) utilizaram para cada constructo que antecede à atitude foram as seguintes:

- Informatividade - Representa o "consenso em relação à capacidade de a propaganda informar aos consumidores sobre as alternativas de produtos" e como consequência, resulta na decisão de compra (Schlosser, Shavitt \& Kanfer, 1999). Ela fornece informações úteis e relevantes para ajudar os consumidores a escolher produtos e serviços que desejam adquirir.

- Entretenimento - Consiste na capacidade de satisfazer e atender às necessidades do coletivo por escapismo, lazer, diversão, prazer, ou liberdade emocional. É determinado como o valor do entretenimento (McQuail, 2010). De acordo com Ducoffe (1996) os consumidores tendem a ter uma apreciação favorável quando assiste a um anúncio divertido.

- Irritação - É a capacidade de "desviar a atenção de objetivos sociais dignos, diluir experiências humanas e explorar a ansiedade humana e as esperanças (Galbraith, Crook, 1958; Shudson, 2013; Yang et al., 2017 p. 843). De acordo com Ducoffe (1996) os consumidores podem ficar irritados com as estratégias de propagandas utilizadas pelas empresas, achando elas chatas, inconvenientes, ofensivas e manipuladoras. Kim, Sundar (2010) enfatizam que a quantidade exagerada de publicidade em sites, e em outros meios, podem causar irritação nos consumidores.

- Credibilidade - Refere-se à confiança que as pessoas têm, ou não, em relação ao conteúdo que contém a propaganda (Brackett \& Carr, 2001; Erkan \& Evans, 2016).

A Teoria do Fluxo e a Teoria da Ação Racional, também integram o modelo para analisar a intenção e o comportamento de compra, neste caso, o comportamento e a intenção do turista perante um vídeo sobre um determinado serviço hoteleiro na internet.

De acordo com Nakamura e Csikszentmihalyi (2009), o fluxo é uma "sensação holística que as pessoas sentem quando agem com total envolvimento" em uma atividade ou em algo. Quando os indivíduos estão em fluxo percebem-se tão envolvidos e absolvidos que nada mais parece ter interesse. Trata-se de um estado de diminuição de "foco da consciência", no qual as percepções e pensamentos desimportantes são selecionados. O fluxo é constituído por oito variáveis, sendo elas: objetivo claro, rápido feedback (interatividade), desafios compatíveis com habilidades, atenção, concentração, sensação de controle, perda da autoconsciência e distorção do tempo (Csikszentmihalyi, 1997). Fluxo é um tipo de concentração mental na navegação na 
internet, especificamente, ao assistir vídeos on-line (Erkan \& Evans, 2016). Por isso, o fluxo é um fator importante para os consumidores, pois aumentam a intenção de compra no comércio on-line (Gao \& Koufaris, 2006; Yang et al., 2016; Yang et al., 2017).

A Teoria da Ação Racional (TRA) foi desenvolvida por Fishbein e Ajzen (1975) e tem o objetivo de entender e predizer o comportamento. De acordo com os autores, essa teoria evidencia que os seres humanos são racionais e usam todas as informações, refletindo sobre suas consequências, para resolver efetivar, ou não, um determinado comportamento. De acordo com Webster, Trevino e Ryan (1994), a TRA dá suporte para assegurar que o comportamento de um indivíduo é estipulado por sua intenção comportamental, e que a intenção comportamental é definida, tanto pela atitude, como pelas normas subjetivas. Para se entender o comportamento é preciso reconhecer o que é determinante das intenções comportamentais, sendo as atitudes de um indivíduo em relação a esse comportamento ou as normas subjetivas referentes a uma determinada influência social. Por exemplo, um grupo de pessoas poderiam persuadir às atitudes (Fishbein \& Ajzen,1975). Já a atitude é um constructo de avaliação positiva ou negativa sobre algo (Webster et al.,1994). E a intenção comportamental está relacionada com a probabilidade de um indivíduo realizar uma ação, e tem efeito direto na sua decisão. A intenção comportamental é à "medida que verifica, se uma pessoa está disposta a tentar e realizar um determinado comportamento" (Fishbein \& Ajzen,1975).

\subsection{Processo Metodológico}

Para entender os fatores que influenciam o comportamento e intenção de compra de serviços hoteleiros ao assistir vídeos na Internet, esta pesquisa parte de uma adaptação metodológica do estudo de Yang et al. (2017), abordando as técnicas adotadas no estudo dos autores, porém, adaptando para uma nova realidade no contexto do setor turístico. Trata-se de um estudo quantitativo, no qual Dencker (2000) explica que se faz necessário a utilização de técnicas e procedimentos estatísticos visando à facilidade de interpretação dos dados de pesquisa. Portanto, as informações são geradas e traduzidas em números, através da análise e interpretação de dados.

Neste contexto, as variáveis testadas fazem parte de teorias que comportam a Teoria da Ação Racional e do Fluxo (Csikszentmihalyi, 1997; Fishbein \& Ajzen, 1975), além das atitudes em relação à publicidade na internet, reunindo as seguintes variáveis: Informatividade, Entretenimento, Irritação e Credibilidade (Galbraith, Crook, 1958; Schlosser, Shavitt \& Kanfer, 
1999; Brackett \& Carr, 2001; McQuail, 2010; Erkan \& Evans, 2016; Shudson, 2013; Yang et al., 2017).

O estudo utilizou um questionário como instrumento para coletar as informações e foi composto por duas fases. A primeira levantou as informações sobre o uso de vídeos a partir da percepção dos participantes. Para isto, ele foi composto por afirmações atribuídas em escala Likert de 5 pontos, variando sobre os níveis de percepções a partir de discordo plenamente até concordo plenamente. As perguntas baseadas no estudo de Yang et al. (2017) passaram por reformulações, primeiro para efetivar um processo de tradução eficaz (já que o estudo original estava no idioma inglês), e depois para adaptar as afirmações para o contexto da percepção de compra de serviços turísticos, mais especificamente, serviços hoteleiros.

Além disso, as perguntas foram alteradas de modo a abranger outras redes sociais e plataformas da internet, uma vez que no estudo original, as perguntas eram direcionadas, especificamente, apenas à plataforma YouTube. Ressalta-se que a estratégia de ampliar para outras redes sociais foi utilizada seguindo uma recomendação estabelecida por Yang et al. (2017), pois de acordo com os autores, apesar do YouTube ser considerada uma das maiores plataformas de vídeos, a velocidade em que ocorrem as inovações e novas redes sociais surgem no mercado, é possível que não só o YouTube, mas também outras redes sociais estejam sendo utilizadas por consumidores de serviços hoteleiros, seja para assistir ou compartilhar vídeos na internet. E por fim, um segundo e último bloco de perguntas incluíram questões demográficas sobre o perfil destes entrevistados.

Os resultados apresentados fazem parte de um estudo piloto realizado durante o mês de abril do ano de 2019. Dencker (2000) explica sobre a importância para o pesquisador realizar um teste piloto para verificar a coerência e efetividade das perguntas. Neste sentido, este estudo piloto foi importante por permitir identificar possíveis falhas ou necessidades de melhorias no instrumento de coleta e adequação das variáveis testadas. Após a divulgação do questionário no formato digital em plataformas na internet, obteve-se um número total de 60 questionários válidos.

Para analisar as informações foram utilizadas, primeiramente uma análise fatorial confirmatória, que teve como objetivo identificar os fatores que poderiam explicar as relações entre um conjunto de variáveis (Corrar, Paulo \& Dias Filho, 2014). Posteriormente, elaborouse uma matriz de correlações com o propósito de analisar relações entre os constructos investigados (Hair, Anderson, Black \& 2009). A análise fatorial e a matriz de correlações foram 
testadas através do software para análise de dados quantitativos Statistical Package for the Social Sciences (SPSS), versão 22.0 para Windows. Portanto, o software foi utilizado para analisar as inter-relações das variáveis do modelo teórico.

\section{Resultados e discussão}

Pelo perfil dos 60 respondentes verifica-se que a maioria são pessoas do gênero feminino $(78,3 \%)$ com idade entre 21 e 40 anos (70\%). Sobre o estado-civil, prevaleceu o número de solteiros (60\%), seguido de casados (30\%). Com relação ao grau de escolaridade, os participantes possuem pelo menos ensino superior completo $(38,3 \%)$ ou pós-graduação completa $(31,7 \%)$. Sobre a renda familiar, a maioria, isto é, 58,4\% apresentaram valores de R\$ 2. 862,00 a $\mathrm{R} \$ 9.540,00$. Enquanto isto, $29,9 \%$ variou entre $\mathrm{R} \$ 954,00$ a $\mathrm{R} \$ 2.862,00$. E 11,7\%

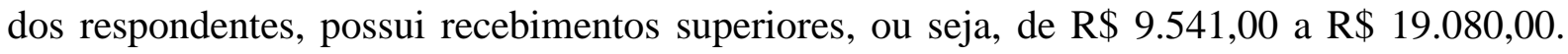
Sobre o local de moradia, os respondentes são residentes dos estados do Rio Grande do Norte, Maceió, Minas Gerais, Rio de Janeiro e Tocantins (Brasil).

Portanto, é possível descrever que a maioria são mulheres, jovens e solteiras, residentes entre as regiões Norte, Nordeste e Sudeste do território brasileiro. Além disto, possuem um bom nível de escolaridade e dispõem de uma renda familiar considerada média e alta. De acordo com a Secretaria de Assuntos Estratégicos (SAE) da presidência da república (2012), é possível classificar a classe média da população brasileira entre aquelas pessoas que recebem valores mensais de 2 a 8 salários mínimos, e a classe alta, acima de 8 salários mínimos, o que vai ao encontro do perfil socioeconômico dos participantes do presente estudo (Valor Econômico, 2019).

Para verificar a adequação das variáveis do modelo foi realizada uma análise fatorial confirmatória de mensuração dos constructos e matriz de correlações. Para examinar o modelo de mensuração foi realizada a verificação da Carga Fatorial, relacionada diretamente com a variável do modelo, da Comunalidade de cada indicador, do Alfa de Cronbach (de cada variável) e por fim, da porcentagem de Variância.

A tabela 01 apresenta os dados referentes à análise fatorial de cada constructo do modelo em termos de Carga Fatorial, Alfa de Cronbach, Comunalidade e Variância. 
Silva, M. M., Silva, J. R. H., Marques Junior, S., \& Mendes Filho, L. (2020). Comportamento do consumidor e intenção de compra de serviços hoteleiros: influência ao assistir vídeos na internet

Tabela 01 - Resultados da análise fatorial

\begin{tabular}{|c|c|c|c|c|c|}
\hline Constructo & $\begin{array}{c}\text { Indicado } \\
\mathbf{r} \\
\end{array}$ & $\begin{array}{c}\text { Carga } \\
\text { fatorial }\end{array}$ & $\begin{array}{c}\text { Alfa de } \\
\text { Cronbach } \alpha\end{array}$ & Comunalidade & $\begin{array}{c}\% \text { de } \\
\text { Variância }\end{array}$ \\
\hline \multirow{3}{*}{ Entretenimento } & ENT1 & 0,933 & \multirow{3}{*}{0,909} & 0,870 & \multirow{3}{*}{84,860} \\
\hline & ENT2 & 0,910 & & 0,828 & \\
\hline & ENT3 & 0921 & & 0,848 & \\
\hline \multirow[t]{3}{*}{ Informatividade } & INF1 & 0,930 & \multirow{3}{*}{0,905} & 0,865 & \multirow{3}{*}{84,144} \\
\hline & INF & 0,943 & & 0,888 & \\
\hline & INF3 & 0,878 & & 0,771 & \\
\hline \multirow[t]{4}{*}{ Irritação } & IRT1 & 0,932 & \multirow{4}{*}{0,954} & 0,868 & \multirow{4}{*}{88,757} \\
\hline & IRT2 & 0,972 & & 0,944 & \\
\hline & IRT3 & 0,924 & & 0,854 & \\
\hline & IRT4 & 0,940 & & 0,884 & \\
\hline \multirow[t]{3}{*}{ Credibilidade } & CRD1 & 0,924 & \multirow{3}{*}{0,924} & 0,853 & \multirow{3}{*}{87,337} \\
\hline & CRD2 & 0,954 & & 0,911 & \\
\hline & CRD3 & 0,925 & & 0,856 & \\
\hline \multirow[t]{3}{*}{ Atitude } & ATI1 & 0,902 & \multirow{3}{*}{0,863} & 0,814 & \multirow{3}{*}{78,786} \\
\hline & ATI2 & 0,841 & & 0,708 & \\
\hline & ATI3 & 0,918 & & 0,842 & \\
\hline \multirow[t]{3}{*}{ Comportamento } & COMP1 & 0,924 & \multirow{3}{*}{0,922} & 0,854 & \multirow{3}{*}{86,490} \\
\hline & COMP2 & 0,932 & & 0,869 & \\
\hline & COMP3 & 0,934 & & 0,872 & \\
\hline \multirow[t]{3}{*}{ Fluxo } & FLU1 & 0,918 & \multirow{3}{*}{0,936} & 0,842 & \multirow{3}{*}{88,756} \\
\hline & FLU2 & 0,968 & & 0,936 & \\
\hline & FLU3 & 0,940 & & 0,884 & \\
\hline \multirow[t]{3}{*}{ Intenção } & INT1 & 0,962 & \multirow{3}{*}{0,957} & 0,925 & \multirow{3}{*}{92,082} \\
\hline & INT2 & 0,956 & & 0,913 & \\
\hline & INT3 & 0,961 & & 0,924 & \\
\hline
\end{tabular}

Fonte: Dados da pesquisa (2019).

A Carga Fatorial pode ser definida como correlações entre as variáveis e os fatores. Quanto maior a Carga Fatorial maior será a correlação com um determinado fator. Cargas fatoriais significativas são com valores acima de 0,5 para explicar o fator de variância (Hair, Anderson \& Black, 2009). A Carga Fatorial de cada indicador testado ficou acima de 0,841. Dessa maneira, pode-se concluir que os indicadores representam bem os constructos apresentados no modelo.

O Alfa de Cronbach $\alpha$ mede a correlação média entre as perguntas. O coeficiente $\alpha$ é calculado a partir da variância dos itens individuais e da variância da soma dos itens de cada avaliador" (Hora, Monteiro \& Arica, 2010, p.89). Valores relevantes de Alfa de Cronbach $\alpha$ são acima de 0,7. Porém, para pesquisas relacionadas às Ciências Sociais Aplicadas, valores acima de 0,6 são aceitos e consideráveis significativos (Hair et al., 2009). Os valores de Alfa de Cronbach $\alpha$ obtidos nesta pesquisa foram superiores a 0,8. Já a porcentagem de Variância deve ser acima de $60 \%$, sendo que a mínima foi de $78 \%$ e a máxima de $92 \%$. O Alfa de 
Cronbach $\alpha$ e a porcentagem de variância foram validadas visto que os limites aceitos foram alcançados (Hair et al., 2009).

Comunalidades são quantidades das correlações de variâncias de cada variável explicada pelos fatores. Quanto maior a Comunalidade maior será o poder de explicação pelo fator. Comunalidades devem ser iguais ou acima de 0,6 para serem consideráveis (Corrar, Paulo \& Dias Filho, 2014). Os dados da pesquisa mostraram que a Comunalidade de cada constructo alcançou valores acima de 0,7 .

Já a porcentagem de Variância é o percentual que explica o quanto a variável tem de significância (Hair et al., 2009). De acordo com Hair et al. (2009), o limite para a porcentagem da Variância considerado satisfatório para explicar o constructo é de 60\%. Todos os itens obtiveram valores acima de $78 \%$, explicando assim o constructo. Em relação à porcentagem de Variância o estudo apresentou resultados significativos.

Diante do exposto, é perceptível que todas as variáveis propostas no modelo obtiveram valores significativos. Os indicadores testados validaram os constructos, uma vez que a Carga Fatorial dos indicadores foram acima de 0,5 e a Comunalidade, dos indicadores, maiores que 0,6. Além disto, todas as cargas fatoriais foram acima de 0,841 (mínima) até 0,972 (máxima). Portanto, obteve-se um bom resultado (Corrar et al., 2014).

Outra análise realizada foi a matriz de correlação, efetuada a partir do coeficiente de Pearson. A correlação de Pearson ("r") é uma medida numérica que verifica e representa a relação de duas variáveis. Em outras palavras, trata-se de um índice que mede o grau de correlação entre as variáveis ou a intensidade da relação (Hair, Anderson \& Black, 2009).

Os coeficientes de Pearson são valores que variam entre $-1,0$ e 1,0 , e reflete na intensidade de uma relação linear entre dois conjuntos de dados. O r=1 (significa uma correlação positiva entre as variáveis); e r=-1(significa uma correlação negativa entre as variáveis); e o r=0 (significa que as variáveis não dependem uma da outra) (Hair, Anderson \& Black, 2009; Corrar, Paulo \& Dias Filho, 2014). O valor de "p" (Pearson) sempre deve estar entre -1 e +1 , se estiver muito próximo de 0 conclui-se que não há uma correlação significativa entre os constructos. E se o $\mathrm{r}$ estiver próximo de -1 ou +1 conclui-se que há uma relação significativa. Porém, deve-se fazer uma análise interpretativa dessa relação.

De acordo com Corrar, Paulo e Dias Filho (2014), se o valor de Pearson (r) estiver entre 0 a 0,3 (positivo ou negativo) a correlação é considerada fraca. Valores entre 0,3 a 0,6 (positivo ou negativo) a correlação é moderada. E valores acima 0,6 (positivo ou negativo) são 
correlações consideradas fortes. A tabela 02 apresenta os dados obtidos na matriz de correlação entre os constructos com seus respectivos valores.

Tabela 02 - Matriz de Correlação

\begin{tabular}{|c|c|c|c|c|c|c|c|c|}
\hline DIMENSÃO & ENTRET & INFO & IRRIT & CRED & ATITUDE & COMPORT & FLUXO & INTENÇÃO \\
\hline ENTRET & 1 & & & & & & & \\
\hline INFO & $0,647^{* *}$ & 1 & & & & & & \\
\hline IRRIT & $-0,498^{* *}$ & $-0,502^{* *}$ & 1 & & & & & \\
\hline CRED & $0,446^{* *}$ & $0,629^{* *}$ & $-0,320^{*}$ & 1 & & & & \\
\hline \begin{tabular}{|l} 
ATITUDE \\
\end{tabular} & $0,586^{* *}$ & $0,751^{* *}$ & $-0,648^{* * *}$ & $0,603^{* *}$ & 1 & & & \\
\hline COMPORT & $0,707^{* *}$ & $0,575^{* *}$ & $-0,434^{* *}$ & $0,495^{* *}$ & $0,666^{* *}$ & 1 & & \\
\hline FLUXO & $0,685^{* *}$ & $0,520^{* *}$ & $-0,410^{* *}$ & $0,534^{* *}$ & $0,596^{* *}$ & $0,725^{* *}$ & 1 & \\
\hline İNTENÇÃO & $0,582^{* *}$ & $0,550^{* *}$ & $-0,434^{* * *}$ & $0,445^{* *}$ & $0,677^{* *}$ & $0,811^{* *}$ & $0,715^{* * *}$ & 1 \\
\hline
\end{tabular}

**. A correlação é significativa no nível 0,01 (2 extremidades).

*. A correlação é significativa no nível 0,05 (2 extremidades).

Fonte: Dados da pesquisa (2019).

A maioria dos resultados foram compatíveis com o estudo de Yang et al. (2017). No entanto, alguns resultados chamaram maior atenção quando aplicados ao contexto do turismo. Verifica-se que assim como ocorreu no estudo de Yang et al. (2017), a correlação da Informatividade com a Atitude obteve o valor de 0,751 , portanto, a correlação foi considerada forte. Logo, percebe-se que a capacidade dos vídeos de gerar informações sobre os produtos e serviços hoteleiros é muito importante e tem relação forte com a atitude do consumidor na tomada de decisão de compra. Este resultado demostra aos gestores de marketing dos hotéis, a importância que existe em disponibilizar informações úteis, não só do estabelecimento hoteleiro em si, mas de outros equipamentos e serviços turísticos disponíveis em seu entorno. Essas informações poderão fazer com que os potenciais clientes tenham uma maior aceitação do vídeo, pois terão a informação de estarem se hospedando em um local que disponibiliza não só a acomodação, mas também, acessibilidade para outros locais que supram suas necessidades básicas enquanto turista.

Vídeos com informações sobre a situação de segurança do destino e da localização em que o hotel se encontra são importantes. De acordo com Amir et al. (2018), essas informações poderão gerar no turista uma menor sensação de risco, direcionando-o a ter a intenção e realizar o comportamento de compra. Tussyadiah e Fesenmaier (2009) também explicam que os vídeos exercem o papel de mediador da experiência turística em fases antecipatórias, durante o planejamento de viagem. Portanto, eles têm o poder de gerar fantasias e prazeres mentais, através do sonho e da imaginação, que fazem com que o turista se desloque de modo a 
experimentar ou vivenciar uma determinada realidade. Ao disponibilizarem informações sobre os equipamentos e acomodações do hotel, ou de lugares turísticos próximos, o empreendimento poderá adquirir uma maior vantagem competitiva frente aos concorrentes, pois os turistas podem sentir um maior desejo e necessidade de utilizar esse estabelecimento, em detrimento de outro.

Outro constructo que também chamou atenção foi a Irritação, pois obteve valores negativos quando correlacionados com todas as outras dimensões. Este resultado vai ao encontro de outros autores (Brackett \& Carr, 2001; Tsang, Ho \& Liang, 2004; Yang et al., 2017), que comprovam seu impacto negativo. Neste contexto, ao analisar a relação da Irritação com a Intenção, assim como da Irritação com o Comportamento, este estudo apresentou valores iguais em ambos os casos, obtendo o valor de -0,434 que significa uma correlação moderada, no entanto negativa. Apesar da correlação moderada entre a Irritação e o Comportamento ter se assemelhado ao estudo de Yang et al. (2017), quando se refere à Irritação com a Intenção, o resultado diverge, visto que o presente estudo apresentou uma correlação ainda mais significativa, se comparado ao estudo dos autores anteriores.

De modo amplo, estes resultados significam que os vídeos podem ser considerados irritantes, ofensivos, hostis, invasivos, e, portanto, os consumidores podem não gostar de assisti-los. Ao se sentir irritado enquanto assiste a um vídeo sobre serviços hoteleiros, o potencial hóspede pode se sentir incomodado e, consequentemente, este incômodo influencie a sua Intenção, levando-o a optar por não exercer o comportamento de compra. Uma alternativa para os gestores diminuírem o sentimento de irritação obtida pelo consumidor, seria por meio de incentivo a formação do CGU em formato de vídeos, pois de acordo com Vargo e Lusch (2016) o consumidor contemporâneo está co-criando com os empreendimentos e entre seus similares, por meio das trocas de informações, compartilhamentos e feedbacks. Portanto, isso contribui com a divulgação e disseminação da marca, mediante seus compartilhamentos e opiniões disponibilizadas na internet. Neste cenário, Silva et al. (2018, p 173), também argumentam que é importante que "os hotéis elaborem estratégias de gerenciamento e incentivo para que os próprios hóspedes realizem publicações em vídeos nos espaços do hotel, e dessa forma, disseminem as informações amplamente entre suas redes de relacionamentos". Essas ações chamarão maior atenção das pessoas, pois diferentemente de uma propaganda massiva realizada de empresa para o consumidor, o vídeo passará a ser divulgado de consumidor para 
consumidor, o que poderá diminuir a irritação em assistir a um vídeo, uma vez que o protagonista do conteúdo certamente será alguém da rede de relacionamento do cliente.

Reino e Hay (2011) também argumentam que uma alternativa seria investir em influenciadores digitais, para divulgar produtos, serviços e destinos turísticos em formato de vídeos. Na percepção dos autores, essa é uma forma de chamar maior atenção das pessoas e despertar maior interesse, devido à popularidade que esses influenciadores possuem perante seus seguidores.

O Entretenimento com o Comportamento obteve uma correlação forte de 0,707. As pessoas que sentem prazer ao assistir um vídeo, também acham agradável e tem atitudes favoráveis que podem gerar intenção de exercer um comportamento de compra. O estudo de Yang et al. (2017) atestaram essa correlação, porém de forma moderada. Isto significa que o entretenimento do vídeo é fundamental para levar o cliente a fechar a reserva no hotel e efetivar a compra. Sobre isto, Silva et al. (2018) também expõem que uma maneira de tornar os vídeos mais interessantes seria por meio de "lives" espontâneas criadas por meio de redes sociais online. Os autores dialogam que estes recursos de marketing digital têm crescido nos últimos anos, e que é importante os gestores de marketing dos hotéis se apropriarem e beneficiar-se deles. Portanto, levando em consideração a espontaneidade, além dos recursos interativos possibilitados por meio das "lives" ao vivo em redes sociais on-line, sugere-se que essa pode ser uma ferramenta em potencial para os hotéis tornar os vídeos mais divertidos, proporcionando o entretenimento e levando ao comportamento de compra.

Outros resultados mostraram que a Atitude com Comportamento alcançou uma correlação forte de 0,666 . Isto significa que as atitudes influenciam o comportamento e quanto mais atitudes positivas e assertivas, maiores as chances e possibilidades das pessoas terem intenção de exercer o comportamento de compra. Se ao assistir um vídeo on-line sobre serviços hoteleiros, o consumidor obtiver confiança e segurança, ele se sentirá apto a realizar e finalizar uma compra. Essa correlação é atestada no estudo de Yang et al. (2017) que também obteve uma correlação forte dessas dimensões. Já a Intenção com o Comportamento apresentou correlação forte, chegando a 0,811. Essa relação mostra que após assistir a um vídeo on-line os consumidores podem ter intenção de comprar um produto ou serviço hoteleiro se o vídeo for agradável, se a experiência for positiva e se a informação for de confiança. Esses fatores contribuem para a atitude do consumidor, que por sua vez, irá aumentar a intenção e acarretar o comportamento. 
Silva, M. M., Silva, J. R. H., Marques Junior, S., \& Mendes Filho, L. (2020). Comportamento do consumidor e intenção de compra de serviços hoteleiros: influência ao assistir vídeos na internet

Já as correlações do Fluxo com a Intenção e do Fluxo com o Comportamento também foram significativamente fortes, de 0,715 e 0,725, respectivamente, obtendo-se novamente resultados semelhantes a Yang et al. (2017). Sendo assim, considera-se que as pessoas podem estar tão envolvidas quando assistem a um vídeo on-line, que todas as informações contidas nele despertam interesse, curiosidade, vontade, e como consequência será capaz de gerar a intenção e o comportamento de compra de serviços hoteleiros.

De modo geral, percebe-se que os resultados da matriz foram de correlações moderadas $(0,3$ e 0,6$)$ e fortes (acima de 0,6$)$. Portanto, as relações entre as dimensões foram significativas e relevantes, com objetivo de explicar a atitude da utilização dos vídeos para intenção de compra de serviços hoteleiros.

\section{Considerações finais}

Pela facilidade e constantes inovações disruptivas dos avanços tecnológicos, percebe-se que tanto os consumidores, quanto os empreendedores, estão fazendo uso de vídeos em seu diaa-dia. Neste sentido, este estudo se propôs a analisar os fatores que influenciam o comportamento e intenção de compra do consumidor de serviços hoteleiros ao assistir vídeos na internet. Para isto, o estudo adaptou-se ao modelo proposto por Yang et al. (2017) que testa as atitudes dos consumidores perante a anúncios de vídeos on-line.

Por meio dos resultados obtidos no estudo piloto constata-se que as correlações testadas foram expressivas, estando entre moderada e alta, o que significa que todos os constructos testados foram confirmados e o modelo validado quando aplicado à realidade dos serviços hoteleiros. Além disso, em alguns casos os valores obtidos foram mais significativos, chamando a atenção, quando comparado com o estudo dos autores precursores (Yang et al., 2017).

A Informatividade e o Entretenimento foram os constructos que mais se relacionaram positivamente. Por outro lado, a Irritação correlacionou-se negativamente com todas dimensões. A Intenção e o Comportamento também estiveram inter-relacionados. Além disso, o estudo confirma que a capacidade de o telespectador entrar em Fluxo durante a exibição de um vídeo, contribui tanto para a Intenção, quanto para o Comportamento de compra.

Por meio dos resultados do estudo, é possível sugerir algumas implicações práticas para serem trabalhadas no contexto desses empreendimentos. Primeiro, sugere-se que os vídeos sejam mais informativos, e para isso, eles devem conter o máximo de informações sobre os serviços do hotel, a segurança do local, além de equipamentos e serviços turísticos disponíveis 
em seu entorno. O uso de vídeos de curta duração também pode ser útil, pois clipes de vídeos podem chamar maior atenção do telespectador, e diminuir possíveis sentimentos de irritação que vídeos duradouros podem provocar (Tsai et al., 2017). Além disso, é importante que os vídeos provoquem entretenimento no telespectador, o que, consequentemente poderá contribuir para minimizar a irritação e elevar a concentração, provocando o estado de Fluxo. Isto pode ser alcançado por meio de "lives" ao vivo e espontâneas criadas pela empresa em redes sociais online, além de estratégias para incentivar a disseminação de conteúdo gerado pelo usuário em forma de vídeos. Assim, estes recursos seriam divulgados de consumidor para consumidor e entre os próprios amigos e conexões do hóspede, ajudando no processo de co-criação do serviço, além de "disfarçar" a principal finalidade dos anúncios que seria a promoção e venda de serviços hoteleiros na internet.

Como este estudo está em processo de desenvolvimento e os resultados fazem parte de um teste piloto, esta pesquisa não contempla percepções de uma ampla amostra de consumidores sobre o uso dos vídeos on-line na intenção de compra para adquirir serviços hoteleiros. Portanto, trata-se de um estudo com dados preliminares, mas que necessita ser ampliado. Neste sentido, é importante que em uma perspectiva futura a pesquisa se amplie para um maior número de respondentes, e que assim, seja possível testar outros procedimentos, tais como: teste de hipóteses e modelagem de equações estruturais. Além disso, também se sugere a aplicação do estudo em outros tipos de segmentos de serviços comercializados dentro da atividade turística.

Por fim, este estudo demonstra a importância do uso de vídeos para a comercialização e venda de serviços hoteleiros, e propõe que os gestores dos hotéis invistam nestes mecanismos para melhorar cada vez mais o seu marketing digital.

\section{Referências}

Administradores. (2017). Estudo aponta as tendências do mercado para os próximos 10 anos. Recuperado em 28 de abril, 2019, de https://administradores.com.br/noticias/estudo-apontaas-tendencias-do-mercado-para-os-proximos-10-anos.

Amir, A. F., Pennington-cinzento, L., Barbe, D. \& Hanafiah, M. H. M. (2018). Exploring National Tourism Organizations (NTOs): use of YouTube to communicate information on destination safety and security, International Jounal of Academic Research in Business \& Social Sciences, 8 (16), 247-266. 
Berthon, P., Pitt, L. \& DesAutels, P. (2011). Unveiling videos: consumer generated ads as qualitative inquiry, Psychology \& Marketing, 28(10), 1044-1060.

Brackett, L.K., \& Carr, B.N. (2001). Cyberspace advertising vs other media: consumer vs mature student attitudes, Journal of Advertising Research, 41(5), 23-32.

Brightcove (2018). Video Marketing Survey. Analyzing the impact that video content has consumers' buying decisions. YouGov Survey. Recuperado em 11 de julho, 2020 de https://investor.brightcove.com/news-releases/news-release-details/new-researchbrightcove-finds-video-content-impacts-product-and.

Csikszentmihalyi, M. (1997). Finding flow. New York: Basic Books.

Corrar, L. J., Paulo, E. \& Dias Filho, J. M. (2014). Análise multivariada para os cursos de administração, ciências contábeis e economia. São Paulo: Atlas.

Dencker, A. F M. (2000). Métodos e técnicas de pesquisa em turismo. 2 ed. São Paulo: Futura.

Díaz Arias, R. (2009). El vídeo en el ciberespacio: usos y lenguaje. Revista Científica de Educomunicació, 33, 63-71.

Ducoffe, R. H. (1996). Advertising value and advertising on the web, Journal of Advertising Research, 36(5), 21-35.

Valor Econômico. (2019). Classes A e B voltam a crescer e atingem 14,4\% da população. Recuperado em 12 de julho de 2020 de https://valor.globo.com/brasil/noticia/2019/10/29/classes-a-e-b-voltam-a-crescer-e-atingem144-da-populacao.ghtml

E-Marketer. (2019). Social vídeo ad spending will grow 44\% by 2021: what's driving marketers to invest in social video. Recuperado em 28 de abril, 2019 de https://www.emarketer.com/content/q1-2019-digital-video-trends.

Erkan, I. \& Evans, C. (2016). The influence of eWOM in social media on consumers' purchase intentions: an extended approach to information adoption, Computers in Human Behavior, 61, 47-55.

Fishbein, M., \& Ajzen, I. (1975). Belief, Attitude, Intention, and Behavior: An Introduction to Theory and Research. Reading, MA: Addison-Wesley.

Galbraith, J. K. \& Crook, A. (1958). The affluent society. Boston: Houghton Mifflin.

Gao, Y. \& Koufaris, M. (2006). Perceptual antecedents of user attitude in electronic commerce, Acm Sigmis Database: The Database for Advances in Information Systems, 37 (2). 42-50. 
Guimarães, A S. \& Borges, M P. (2008). E-turismo: internet e negócios do turismo. São Paulo: Cengage Learning.

Hair Jr., J. F., Anderson, R. E. \& Black, W. C. (2009). Análise multivariada de dados. $6^{\mathrm{a}}$ ed. Porto Alegre: Bookman.

Hora, H. R., Monteiro, G. T. R. \& Arica, J. (2010). Confiabilidade em questionários para qualidade: um estudo com o coeficiente alfa de cronbach, Produto \& Produção, 11(2), 85103.

Hsu, C. L. \& Lu, H. P. (2004). Why do people play on-line games? an extended TAM with social influences and flow experience, Information \& Management, 41(7), 853-868.

Koufaris, M. (2002). Applying the technology acceptance model and flow theory to online consumer behavior, Information Systems Research, 13(2), 205-223.

Litvin, S. W., Goldsmith, R. E., \& Pan, B. (2008). Electronic word-of-mouth in hospitality and tourism management. Tourism Management, 29 (3), 458-468.

Litvin, S. W., Goldsmith, R. E., \& Pan, B. (2018). A retrospective view of electronic word-ofmouth in hospitality and tourism management. International Journal of Contemporary Hospitality Management, 30(1), 313-325.

Mendes-Filho, L., Mills, A. M., Tan, F. B. \& Milne, S. (2018). Empowering the traveler: an examination of the impact of user-generated content on travel planning, Journal of Travel \& Tourism Marketing, 35(4), 425-436.

Mendes Filho, L. (2014). Empowerment in the context of User-Generated Content in the Travel Industry: A research model proposal. El Periplo Sustentable, 27, 4-20.

McQuail, D. (2010). McQuail's Mass Communication Theory. Sage Publications.

Murillo, E., Merino, M. \& Núñez, A. (2016). The advertising value of Twitter ads: a study among mexican millennials, Review of Business Management, 18(61), 436-456.

Nakamura, J., \& Csikszentmihalyi, M. (2009). The concept of flow. In Snyder, C. R., \& Lopez, S. J. (Ed.). Oxford handbook of positive psychology. Oxford University Press, 89105.

Ogden, J. R. \& Crescitelli, E. (2007). Comunicação integrada de marketing: conceitos, técnicas e práticas. 2 ed. São Paulo: Pearson.

Ramos, A., Mendes Filho, L. A. M., \& Lobianco, M. M. (2017). Sistemas e tecnologia da informação no turismo: um enfoque gerencial. Curitiba: Prismas.

Reino, S. \& Hay, B. (2011). The use of YouTube as a tourism marketing tool. Proceedings of the 42nd Annual Travel \& Tourism Research Association Conference, London, Ontario, Canada, 42. 
Ruiz, F. J. M. \& Ortiz Sobrino, M. A. (2016). El vídeo como soporte en la narrativa digital del branded content y los productos audiovisuales en las plataformas online. Poliantea, 12(22), 85-116.

Schlosser, A. E., Shavitt, S. \& Kanfer, A. (1999). Survey of internet users' attitudes toward internet advertising, Journal of Interactive Marketing, 13(3), 34-54.

Schudson, M. (2013). Advertising, the uneasy persuasion (RLE Advertising): its dubious impact on American society. Routledge.

Silva, M. M., Silva, J. R. H. \& Mendes Filho, L. (2018). Uso de vídeos como elemento de promoção e venda de serviços hoteleiros na internet. In: Ramos, C. et al. (Org.). O futuro hoje: novas tecnologias, social media e negócio digital. Faro, Portugal: Universidade do Algarve.

Silva, M. M., Mendes Filho, \& Marques Júnior, S. (2019). Comentarios de viajes online sobre médios de hospedaje: Un abordaje cuantitativo con viajeros de la ciudad de Natal (Brasil). Estudios y Perspectivas en Turismo, 28(1), 185 - 206

Tsai, F. M., \& Bui, T. D. (2020). Impact of word of mouth via social media on consumer intention to purchase cruise travel products. Maritime Policy and Management, 1-17.

Tsai, Y. C., Chu, C. M \& Kabori, K. (2017). The influence of video clips on travel intention and destination image, International Journal of Arts and Commerce, 6(1), 37-55.

Tsang, M. M., Ho, S. C. \& Liang, T. P. (2004). Consumer attitudes toward mobile advertising: an empirical study", International Journal of Electronic Commerce, 8(3), 65 78 .

Tussyadiah, L, P. \& Fesenmaier, D, R. (2009). Mediating tourist experiences: access to places via shared videos, Annals of Tourism Research, 36(1), 24-40.

Vargo, S. L., \& Lusch, R. F. (2016). Institutions and axioms: an extension and update of service-dominant logic. Journal of the Academic Marketing Science, 44,5-23.

Webster, J., Trevino, L. K., \& Ryan, L. (1994). The dimensionality and correlates of flow in human - computer interactions, Computers in Human Behavior, 9(4), 411-426.

Yan, Q., Wu, S., Wang, L., Wu, P., Chen, H. \& Wei, G. (2016). E-WOM from e-commerce websites and social media: which will consumers adopt? Electronic Commerce Research and Applications, 17, 62-73.

Yan, Q., Zhou, S., \& Wu, S. (2018). The influences of tourists' emotions on the selection of electronic word of mouth platforms. Tourism Management, 66, 348-363.

Yang, K.C., Huang, C. H., Yang, C. \& Yang, S. Y. (2017). Consumer attitudes toward online video advertisement: YouTube as a platform, Kybernetes, 46(5), 840-853. 